\title{
Population pharmacokinetic modelling to quantify the magnitude of drug-drug interactions between amlodipine and antiretroviral drugs
}

\author{
Perrine Courlet ${ }^{1}$ (D) Monia Guidi ${ }^{1,2,3}$ (D) Susana Alves Saldanha ${ }^{1} \cdot$ Matthias Cavassini $^{4}\left(\mathbb{D} \cdot\right.$ Marcel Stoeckle $^{5}$. \\ Thierry Buclin $^{1}$ (I) $\cdot$ Catia Marzolini $^{5}$ (1) $\cdot$ Laurent A. Decosterd $^{1}$ (I) $\cdot$ Chantal Csajka $^{2,3,6}$ (1)
}

Received: 17 September 2020 / Accepted: 26 November 2020 / Published online: 16 January 2021

(C) The Author(s) 2020

\begin{abstract}
Purpose Drug-drug interactions (DDIs) with antiretroviral drugs (ARVs) represent an important issue in elderly people living with HIV (PLWH). Amlodipine is a commonly prescribed antihypertensive drug metabolized by CYP3A4, thus predisposed to a risk of DDIs. Guidance on the management of DDIs is mostly based on theoretical considerations derived from coadministration with other CYP3A4 inhibitors. This study aimed at characterizing the magnitude of DDIs between amlodipine and ARV drugs in order to establish dosing recommendations.

Methods A population pharmacokinetic analysis was developed using non-linear mixed effect modelling (NONMEM) and included 163 amlodipine concentrations from 55 PLWH. Various structural and error models were compared to characterize optimally the concentration-time profile of amlodipine. Demographic and clinical characteristics as well as comedications were tested as potential influential covariates. Model-based simulations were performed to compare amlodipine exposure (i.e. area under the curve, AUC) between coadministered ARV drugs.

Results Amlodipine concentration-time profile was best described using a one-compartment model with first-order absorption and a lag-time. Amlodipine apparent clearance was influenced by both CYP3A4 inhibitors and efavirenz (CYP3A4 inducer). Model-based simulations revealed that amlodipine AUC increased by $96 \%$ when coadministered with CYP3A4 inhibitors, while efavirenz decreased drug exposure by $59 \%$.

Conclusion Coadministered ARV drugs significantly impact amlodipine disposition in PLWH. Clinicians should adjust amlodipine dosage accordingly, by halving the dosage in PLWH receiving ARV with inhibitory properties (mainly ritonavirboosted darunavir), whereas they should double amlodipine doses when coadministering it with efavirenz, under appropriate monitoring of clinical response and tolerance.
\end{abstract}

Keywords Amlodipine $\cdot$ Pharmacokinetics $\cdot$ Drug-drug interactions $\cdot$ HIV $\cdot$ NONMEM

\section{Introduction}

The advent of antiretroviral therapies (ARVs) in the 1990s has revolutionized HIV care, now considered as a fairly

Chantal Csajka

chantal.csajka@chuv.ch

1 Service of Clinical Pharmacology, Lausanne University Hospital and University of Lausanne, Lausanne, Switzerland

2 Centre for Research and Innovation in Clinical Pharmaceutical Sciences, Lausanne University Hospital and University of Lausanne, Lausanne, Switzerland manageable chronic condition. Meanwhile, the management of HIV infection is becoming more challenging, with an ageing HIV-infected population increasingly affected by physiological changes and age-related

3 Institute of Pharmaceutical Sciences of Western Switzerland, University of Geneva, University of Lausanne, Geneva, Switzerland

4 Service of Infectious Diseases, Lausanne University Hospital and University of Lausanne, Lausanne, Switzerland

5 Departments of Medicine and Clinical Research, University Hospital of Basel and University of Basel, Basel, Switzerland

6 School of Pharmaceutical Sciences, University of Geneva, Geneva, Switzerland 
comorbidities. People living with HIV (PLWH) are predisposed to the risk of polypharmacy, thus increasing the burden of drug-drug interactions (DDIs). Indeed, ARV drugs are among therapeutic agents with the highest potential for DDIs, due to the frequent inhibition or induction of several cytochrome P450 (CYP) isoforms.

Despite its predisposition to be victim of DDIs with ARV drugs, amlodipine is a calcium channel blocker commonly prescribed in PLWH; this is probably explained by its rather large therapeutic margin. It is a molecule predominantly metabolized by CYP3A4/5 [1, 2], still with a lower hepatic extraction than other dihydropyridine calcium antagonists [3]. Nevertheless, it might be subject to potential DDIs with strong CYP3A4 inhibitors such as darunavir/ritonavir or with inducers such as efavirenz. However, the magnitude of DDIs with ARV drugs remained to be explored. Although the simple monitoring of blood pressure allows the evaluation of the clinical response to amlodipine, the knowledge of the magnitude of DDIs could guide drug dosage, taking into account the concomitant ARV treatment. The summary of product characteristics of amlodipine mentions an increase in amlodipine exposure $(+60 \%)$ in the presence of diltiazem, a CYP3A4 inhibitor [4]. The label also indicates that a more pronounced increase is expected with other strong inhibitors like ritonavir, without further details on the magnitude of the interaction, neither with guidance on how to adjust amlodipine dosage. Moreover, DDIs between amlodipine and several antiviral agents for the treatment of chronic hepatitis $\mathrm{C}$ infection or firstgeneration ARV drug (e.g. ritonavir-boosted indinavir, which is no longer prescribed) have been evaluated using non-compartmental analyses $[1,5]$. Authors demonstrated a 2-fold increase in amlodipine exposure when coadministered with these drugs, suggesting halving the dosage in individuals receiving such regimens. However, available data on DDIs were mostly collected in healthy young volunteers and therefore may not fully reflect the complex real-life situation of elderly PLWH.

Published population pharmacokinetic (PK) models have investigated amlodipine clearance in different populations (i.e. healthy volunteers, children, adolescents, patients living in nursing homes) and identified body weight, gender and age as the most important factors accounting for pharmacokinetic heterogeneity [6-10]. However, to our knowledge, no population PK models have been developed for amlodipine in PLWH.

The objectives of this study were thus to develop a population PK model of amlodipine in ageing PLWH and to perform model-based simulations to compare amlodipine exposure between various concomitantly prescribed ARV regimens, thus allowing the establishment of reliable dosage recommendations.

\section{Methods}

\section{Study design and participants}

Plasma samples were collected in PLWH followed up in Lausanne and Basel, in the framework of a prospective Swiss HIV Cohort Study project \#815 designed to evaluate clinically significant DDIs between ARVs and frequently prescribed comedications. In addition, PLWH participating in the pharmacokinetic study NCT03515772 (registered in clinicaltrials.gov) contributed with intensive sampling. Study participants gave written informed consent before entering the study. The study protocol was reviewed and approved by the Ethics Committee of Vaud and northwest/central Switzerland (CER-VD 2018-00369).

Undetectable amlodipine plasma concentrations, suggestive of non-adherence to treatment, or samples with missing information about drug administration, sampling times or dosing schedule were excluded from the analysis. For all PLWH, age, bodyweight, gender, liver function tests (aspartate aminotransferase (AST), alanine aminotransferase (ALT), albumin), creatinine clearance (calculated with the Cockroft and Gault formula [11]), and comedications (HIV and non-HIV medications) were recorded.

\section{Plasma concentration determination}

All blood samples were collected and centrifuged in EDTAcontaining tubes. Plasma samples were stored at $-80^{\circ} \mathrm{C}$ until batch analysis using an ultra-high-performance liquid chromatography tandem mass spectrometry (UHPLC-MS/MS) method [12]. Plasma samples underwent protein precipitation with methanol, followed by evaporation of the supernatant at room temperature under nitrogen. The lower limit of quantification was $0.3 \mathrm{ng} / \mathrm{mL}$, well below trough concentrations commonly observed in clinical practice $[9,13,14]$.

\section{Model-based pharmacokinetic analysis}

\section{Structural and error model}

A population PK analysis was performed using non-linear mixed effect modelling (NONMEM v7.3, ICON Development Solutions, Ellicott City, MD, USA) to characterize amlodipine concentration-time profile in PLWH. PsN v4.2.0 was used for automation of model development and evaluation, Pirana v2.9.2 for structure model development and R v3.3.1 (Rstudio v.1.1.423) for data management, statistical analysis and graphical output $[15,16]$. One- and twocompartment disposition models were compared, while evaluating various modelling options for the absorption phase: zero-, first-order or combined zero- and first-order absorption, with or without lag-time, or transit compartments models. 
Between-subject variability (BSV) was described by exponential errors following a log-normal distribution with mean zero and variance $\omega^{2}$, expressed ad coefficient of variation (CV). Several error models (i.e. proportional, additive and mixed) were compared to describe the residual variability.

\section{Covariate model}

First, the correlations between post hoc individual estimates of the PK parameters and the available biologically plausible covariates were inspected visually. Covariates considered as potentially influent were then sequentially included into the model using a stepwise insertion/deletion approach. ARV drugs were classified as strong CYP3A4 inhibitors (i.e. ritonavir-boosted darunavir, cobicistat-boosted darunavir, ritonavir-boosted atazanavir, cobicistat-boosted elvitegravir) or moderate inducers (i.e. efavirenz, etravirine), according to the lists published by the FDA [17]. The effect of the weaker CYP3A4 inducer nevirapine was also tested. Linear or nonlinear functions were used as appropriate (categorical variables coded as 0 and 1 and continuous variables centred on their median values). Missing values for continuous covariates were imputed to the population median value.

\section{Model selection and parameter estimation}

Amlodipine concentration-time profiles were fitted using the first-order conditional estimation method with interaction (FOCEI). Discrimination between hierarchical models was based on the variation of the objective function value ( $\triangle \mathrm{OFV},-2 \log$ likelihood, approximate chi-square distribution) using the likelihood ratio test. For one additional parameter, a decrease of $\triangle$ OFV exceeding $3.84(p<0.05)$ or $6.63(p<0.01)$ was considered statistically significant during model building and backward deletion steps, respectively. Reliability of the results was evaluated using diagnostic plots, along with precision of pharmacokinetic parameters estimates and eta-shrinkage.

\section{Model evaluation and assessment}

The bootstrap method implemented in PsN was employed to validate the stability and performance of the final population PK model, using 2000 bootstrap samplings with replacement [15]. Median parameter values with their $95 \%$ confidence interval $\left(\mathrm{CI}_{95 \%}\right)$ generated by bootstrap were compared with the original model estimates. The predictive performance of the final pharmacokinetic model was evaluated with the normalized prediction distribution errors (NPDEs). In addition, prediction-corrected visual predictive check (pcVPC) was performed.

\section{Model-based simulations}

Amlodipine maximum $\left(\mathrm{C}_{\max }\right)$ and trough concentrations $\left(\mathrm{C}_{\text {trough }}\right)$, along with area under the curve from time 0 to $24 \mathrm{~h}\left(\mathrm{AUC}_{0-24}\right)$, were computed in 1000 simulated PLWH per different ARV regimen (CYP3A4 inhibitors, efavirenz or ARVs devoid of interaction potential with amlodipine). Average $\mathrm{C}_{\text {max }}, \mathrm{C}_{\text {trough }}$ and $\mathrm{AUC}_{0-24}$ between ARV groups were compared.

\section{Results}

\section{Data}

A total of 163 amlodipine concentrations were available from 55 PLWH, eight of whom participated in the PK study with rich sampling and provided 84 concentrations. PLWH in the SHCS\#815 project provided a median (range) of one sample (1 to 3 ) while the median was 11 (8 to 11 ) for individuals included in the rich PK study. Amlodipine was administered at dosages ranging from 2.5 to $10 \mathrm{mg}$ once daily (qd). Three PLWH received amlodipine $5 \mathrm{mg}$ twice daily (bid). The measured plasma concentrations varied from 0.4 to $70 \mathrm{ng} / \mathrm{mL}$. Steady state was assumed for all PLWH. The characteristics of the study population are presented in Table 1. Ritonavirboosted darunavir was the most frequently prescribed ARV with CYP3A4 inhibitory properties.

\section{Structural, statistical and covariate models}

A one-compartment model provided the best fit for amlodipine disposition, while a first-order process adequately described absorption. The addition of an absorption lag-time (ALAG) significantly improved the fit $(\triangle \mathrm{OFV}=-7.39, p=0.007)$.

BSV needed to be assigned only on clearance, as the addition of BSV on the other parameters did not improve data description $(\triangle \mathrm{OFV}>-1.84, p>0.17)$. The population estimates of the PK parameters with the base model were an absorption rate constant $\left(\mathrm{k}_{\mathrm{a}}\right)$ of $0.66 \mathrm{~h}^{-1}$, an ALAG of $0.86 \mathrm{~h}$, a volume of distribution of $980 \mathrm{~L}$ and a clearance of $15.7 \mathrm{~L} / \mathrm{h}$, with a $\mathrm{CV}$ of $61 \%$ characterizing its BSV.

An additive error model adequately described the residual (intra-patient) variability. Univariate analyses revealed clear effects of the CYP3A4 inhibitors $(\triangle \mathrm{OFV}=-20.9$, $p<0.001)$ and of efavirenz $(\Delta \mathrm{OFV}=-10.8, p=0.001)$ on amlodipine elimination, with clearance decreasing by $49 \%$ and increasing by $40 \%$, respectively. Both these covariates explained together $48 \%$ of the variance on clearance [18]. In contrast, we did not identify significant effects for age, sex, weight, albumin, AST, ALT and creatinine clearance on amlodipine disposition $(\Delta \mathrm{OFV}>-1.1, p>0.29)$. Finally, 
Table 1 Demographic and clinical characteristics of PLWH included in the model development dataset

\begin{tabular}{|c|c|}
\hline Patients' characteristics $(N=55)$ & Median $[\mathrm{IQR}]$ or $n(\%)$ \\
\hline Age (years) & $61[53-70]$ \\
\hline Male sex & $41(75)$ \\
\hline Body weight (kg) & 79 [71-91] \\
\hline Missing data & $9(16)$ \\
\hline Systolic blood pressure (mmHg) & $140[130-150]$ \\
\hline Missing data & $9(16)$ \\
\hline Diastolic blood pressure (mmHg) & 83 [75-93] \\
\hline Missing data & $9(16)$ \\
\hline ALT (IU/L) & $27[20-41]$ \\
\hline Missing data & $11(20)$ \\
\hline AST (IU/L) & $26[20-35]$ \\
\hline Missing data & $11(20)$ \\
\hline Albumin $(\mathrm{g} / \mathrm{L})$ & $42[41-44]$ \\
\hline Missing data & $11(20)$ \\
\hline Creatinine clearance (mL/min) & $82[50-101]$ \\
\hline Missing data & $17(31)$ \\
\hline Comedications $(N=163)$ & $n(\%)$ \\
\hline \multicolumn{2}{|l|}{ CYP3A4 inhibitors } \\
\hline Ritonavir-boosted darunavir & $27(17)$ \\
\hline Cobicistat-boosted darunavir & $1(1)$ \\
\hline Ritonavir-boosted atazanavir & $1(1)$ \\
\hline Cobicistat-boosted elvitegravir & $7(4)$ \\
\hline \multicolumn{2}{|l|}{ CYP3A4 inducers } \\
\hline Efavirenz & $7(4)$ \\
\hline Etravirine & $18(11)$ \\
\hline \multicolumn{2}{|l|}{ Others } \\
\hline Nevirapine & $8(5)$ \\
\hline Rilpivirine & $2(1)$ \\
\hline Dolutegravir & $103(63)$ \\
\hline Raltegravir & $8(5)$ \\
\hline Anti-hypertensive agents & $137(84)$ \\
\hline
\end{tabular}

coadministration of ritonavir-boosted darunavir in two patients (13 amlodipine concentrations) receiving etravirine prevented the estimation of the effect of etravirine on amlodipine disposition.

\section{Model evaluation}

The final PK parameter estimates are summarized in Table 2, along with their bootstrap estimations. Shrinkage was equal to $9 \%$ for BSV on clearance and to $15 \%$ for residual variability. All estimates lied within the bootstrap $\mathrm{CI}_{95 \%}$ and differed by less than $7 \%$ from the median bootstrap value, except for ka $(17 \%)$, supporting the reliability of the model. Goodness of fit plots are presented in supplementary material 1 . Normalized prediction distribution errors were not found to significantly differ from a normal distribution. As shown in Fig. 1, pcVPC confirmed the good predictive performance of the model.

\section{Model-based simulations}

Simulations showed a $96 \%$ increase and a $59 \%$ decrease of amlodipine $\mathrm{AUC}_{0-24}$ in patients taking the initial recommended dosage of $5 \mathrm{mg}$ of amlodipine qd with CYP3A4 inhibitors and efavirenz, respectively, compared to amlodipine at the standard dosage alone.

Figure 2 compares amlodipine concentration-time profiles under the standard posology with alternative amlodipine dosage regimens in the presence of CYP3A4 inhibitors or efavirenz. The predicted concentration-time profile of $2.5 \mathrm{mg}$ of amlodipine with CYP3A4 inhibitors almost entirely overlaps with the curve of $5 \mathrm{mg}$ qd alone ( $8 \%$ and $2 \%$ decrease in $\mathrm{C}_{\max }$ and $\mathrm{AUC}_{0-24}$, respectively, while $\mathrm{C}_{\text {trough }}$ increases by $8 \%$ in the alternative vs standard regimen, Table 3). On the other hand, the dosage of $10 \mathrm{mg}$ qd in the presence of efavirenz seems to provide slightly lower exposure $\left(\mathrm{C}_{\max }\right.$ increased by $1 \%, \mathrm{C}_{\text {trough }}$ and $\mathrm{AUC}_{0-24}$ decreased by $38 \%$ and $17 \%$, respectively, in the alternative vs standard regimen, Table 3). Increasing the dose to $15 \mathrm{mg}$ qd in presence of efavirenz would result in a $51 \%$ and $25 \%$ increase in $\mathrm{C}_{\max }$ and AUC, respectively, and a $7 \%$ decrease in $\mathrm{C}_{\text {trough. }}$. A twice daily regimen of amlodipine when coadministered with efavirenz (i.e. $5 \mathrm{mg}$ bid) would decrease $\mathrm{C}_{\text {trough }}, \mathrm{C}_{\max }$ and $\mathrm{AUC}_{0-24}$ by $15 \%, 17 \%$ and $16 \%$, respectively. These alternative dosage regimens (i.e. $15 \mathrm{mg}$ qd and $5 \mathrm{mg}$ bid) in presence of efavirenz are presented in supplementary materials 2 and 3 .

None of the PLWH receiving CYP3A4 inhibitors was concomitantly treated with efavirenz.

\section{Discussion}

This study aimed to investigate the magnitude of DDIs between amlodipine and ARV drugs. For this purpose, the inhibitory or inducing potential of ARVs on amlodipine disposition was assessed in Swiss PLWH enrolled in two pharmacokinetic studies. The pharmacokinetic parameters of amlodipine alone are in good agreement with reported values, still with a lower apparent clearance in our analysis [6-9]. Several studies used a 2-compartment model to describe amlodipine concentration-time profile [7, 10, 19]. The sampling design of our study limited to $28 \mathrm{~h}$ after the last drug intake prevented us to capture adequately the prolonged terminal elimination phase of the drug [20]. However, the halflife calculated using our parameter estimates $(40.8 \mathrm{~h})$ was in accordance with the manufacturer's data ( 35 to $50 \mathrm{~h}$ ), and our model was thus considered adequate. Despite the important BSV, none of the tested demographic covariates showed any 
Table 2 Final population parameter estimates of amlodipine with the bootstrap results. $k_{a}$ first-order absorption rate constant, $A L A G$ absorption lag-time, $C L$ mean apparent amlodipine clearance, $V$ mean apparent volume of distribution, $C I_{95 \%} 95 \%$ confidence interval, $C Y P$ cytochrome $\mathrm{P} 450, C V s$ coefficients of variation, RSES relative standard errors defined as SE/ estimate and expressed as percentages. CYP3A4 inhibitors included ritonavir-boosted darunavir, cobicistat-boosted darunavir, ritonavir-boosted atazanavir and cobicistat-boosted elvitegravir.

\begin{tabular}{|c|c|c|c|c|}
\hline \multirow[b]{2}{*}{ Parameters } & \multicolumn{2}{|c|}{ Final pharmacokinetic model } & \multicolumn{2}{|c|}{$\begin{array}{l}\text { Bootstrap } \\
(n=2000 \text { samples })\end{array}$} \\
\hline & Estimate & $\operatorname{RSE}(\%)$ & Median & $\mathrm{CI}_{95 \%}$ \\
\hline $\mathrm{k}_{\mathrm{a}}\left(\mathrm{h}^{-1}\right)$ & 0.69 & 25 & 0.80 & $0.44-2.15$ \\
\hline ALAG (h) & 0.87 & 28 & 0.90 & $0.24-2.41$ \\
\hline $\mathrm{V} / F(\mathrm{~L})$ & 1000 & 16 & 985 & $777-1472$ \\
\hline $\mathrm{CL} / F(\mathrm{~L} / \mathrm{h})$ & 17.0 & 9 & 16.9 & $14.6-20.5$ \\
\hline $\mathrm{BSV}_{\mathrm{CL}}(\mathrm{CV} \%)$ & 42 & 19 & 40 & $27-59$ \\
\hline$\theta_{\mathrm{CYP} 3 \mathrm{~A} 4 \text { inhibitors }}$ & -0.49 & 12 & -0.49 & -0.60 to -0.34 \\
\hline$\theta_{\text {efavirenz }}$ & 1.40 & 37 & 1.46 & $0.55-3.35$ \\
\hline$\sigma_{\text {add }}(\mathrm{ng} / \mathrm{mL})$ & 2.85 & 11 & 2.77 & $2.17-3.49$ \\
\hline
\end{tabular}

Final model: $\mathrm{CL} / F=17.0 \times(1-0.49 \times \mathrm{CYP} 3 \mathrm{~A} 4$ inhibitors $) \times(1+1.40 \mathrm{x}$ efavirenz $)$ significant influence on the pharmacokinetics of amlodipine that would support dosage adjustment recommendations. In the present study however, we observe a $49 \%$ decrease in amlodipine clearance in case of coadministration of CYP3A4 inhibitors (mainly ritonavir-boosted darunavir). This result is in good agreement with a pharmacokinetic study conducted in healthy volunteers, where amlodipine exposure was increased by $90 \%$ when coadministered with ritonavirboosted indinavir [1]. In addition, two physiologically based pharmacokinetic models reported a 2-fold increase in amlodipine AUC when coadministered with ritonavir [21, 22]. Two other studies showed a more pronounced increase in amlodipine exposure when coadministered with anti-HCV agents (2.6-fold increase in amlodipine AUC with ombitasvir/

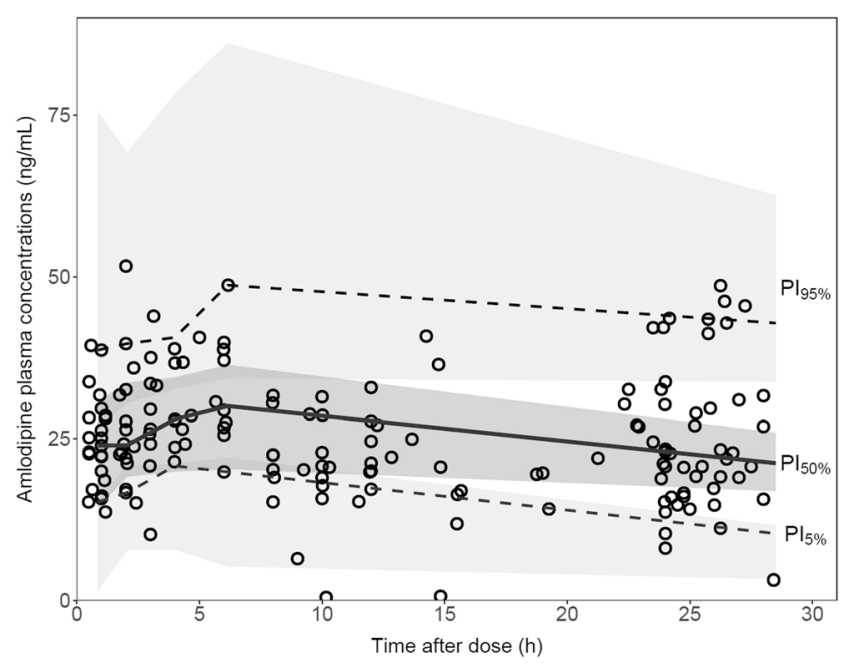

Fig. 1 pcVPC of amlodipine final model with amlodipine predictioncorrected concentrations (open circles), median of the observed concentrations (solid line) with $90 \%$ prediction interval (dashed lines). Grey fields represent the model-based $90 \%$ confidence interval of the simulated median and $\mathrm{PI}_{90 \%}$ paritaprevir/ritonavir/dasabuvir and 2.8-fold increase with telaprevir $[2,23])$. Accordingly, the "DDI-predictor" webtool predicts an AUC ratio (AUC with interactor/AUC without interactor) of 2.7 based on several pharmacokinetic studies [24]. Using a similar approach, Stader et al. demonstrated a 90\% increase in amlodipine exposure when coadministered with ritonavir [25]. Finally, the magnitude of DDI is not expected to differ when amlodipine is coadministered with ritonavir or cobicistat as these PK boosters are equally strong inhibitors of CYP3A4 that is responsible for amlodipine metabolism. However, differences in the magnitude of DDIs could occur for drugs whose metabolism involves CYP2B6, CYP2C9, CYP2C19, CYP1A2 or UGT since ritonavir induces these enzymes whereas cobicistat does not [26]. While the magnitude of the DDI between amlodipine and CYP3A4 inhibitors varies between studies, our results supports the proposed 50\% reduction in amlodipine dosage in case of coadministration with ritonavir-boosted darunavir [27].

Although no upper tolerability threshold has been established for plasma concentrations, an increase in amlodipine exposure is not devoid of clinical consequences. Indeed, serious adverse events such as severe hypotension, oedema and bradycardia have been reported in PLWH receiving both calcium-channel blockers and ARVs with inhibitory potential [28-31]. This suggests caution when prescribing amlodipine in elderly PLWH receiving boosted ARVs, also considering pharmacokinetic and pharmacodynamic interactions with comedications and comorbidities.

To the best of our knowledge, coadministration of amlodipine and ARVs with enzyme inducing properties has not been studied until now. Our results demonstrate a 59\% decrease in amlodipine exposure when coadministered with efavirenz. The lack of plasma concentrations from PLWH receiving etravirine alone prevented the estimation of the 

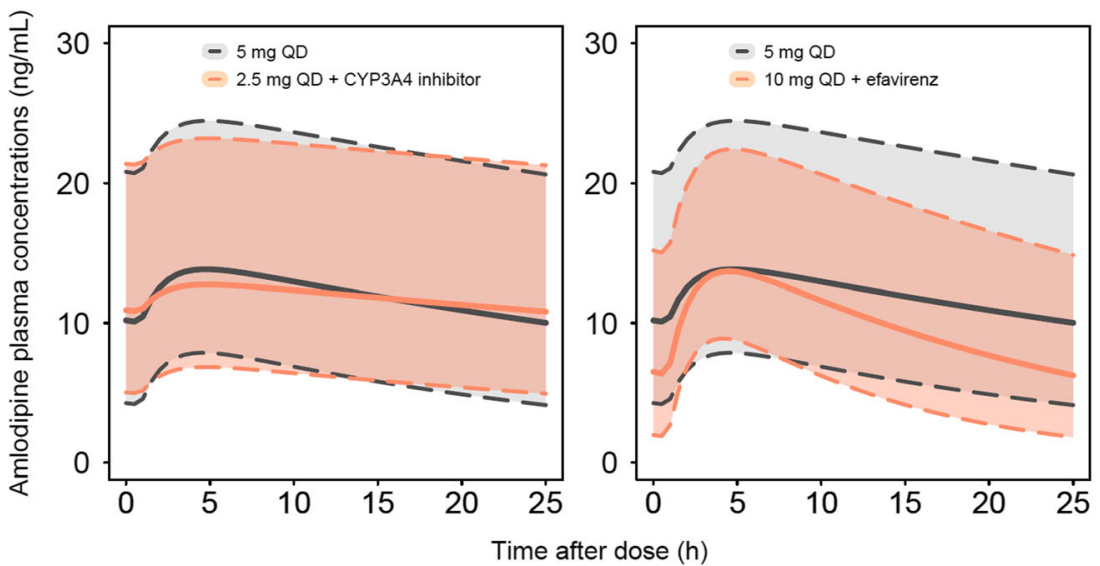

Fig. 2 Simulated amlodipine plasma concentrations for dosage regimens of $2.5 \mathrm{mg}$ qd with CYP3A4 inhibitors (i.e. ritonavir-boosted darunavir, cobicistat-boosted darunavir, ritonavir-boosted atazanavir, cobicistatboosted elvitegravir) (left) or $10 \mathrm{mg}$ qd with efavirenz (right), compared

effect of etravirine on amlodipine PK. The inducing potency of etravirine is possibly lower than that of efavirenz, as observed, for example, on erlotinib and gefitinib (both CYP3A4 substrates) [32], although controversies persist about the respective inducing potential of etravirine and efavirenz [25, 32]. Pharmacokinetic-pharmacodynamic relationships have been established, indicating an impact of amlodipine plasma concentrations on antihypertensive effect [7,9]. In case of treatment initiation, induction can take approximately 10 days to achieve its maximal effect, as reported for others CYP3A4 inducers [33]. However, the time course of CYP3A4 induction seems to be of limited clinical relevance in the case of amlodipine, prescribed for the long-term treatment of the chronic conditions of the patients included in our study. In addition, particular attention must be paid to elderly PLWH as they might be more sensitive to the effect of amlodipine given that the arterial baroreflex function is altered in elderly individuals [34]. Our results support the suggestion that amlodipine dosage should be doubled when coadministered with efavirenz, to reach a plasma exposure comparable to with standard dosage of $5 \mathrm{mg}$ qd. Continuous line represent the population median prediction for standard (grey) and alternative regimens (orange), while shaded areas and dashed lines represent the $90 \%$ prediction interval based on 1000 simulated PLWH

individuals not receiving interacting drugs, and therefore a similar antihypertensive effect. A bid regimen can also be proposed but lower adherence has been reported in PLWH receiving such an ARV regimen compared to a qd dosage [35]. In addition, PLWH from the Swiss HIV Cohort Study have lower adherence to their comedications in comparison to ARV [36]. Thus, a double dose in a qd regimen should be preferred over a bid regimen. This is also supported by the similar $\mathrm{AUC}_{0-24}$ (representing the global exposure) using a $10 \mathrm{mg}$ qd regimen or a $5 \mathrm{mg}$ bid regimen, both in presence of efavirenz. Obviously, amlodipine dosage decisions should rest on the global evaluation of the patient's condition and response to treatment.

We have no observations of amlodipine exposure when coadministered with both CYP3A4 inhibitors and efavirenz. One PLWH included in the PK study with rich sampling received both ritonavir-boosted darunavir and etravirine, and had rather high amlodipine plasma concentrations. This indicates a predominance of the CYP3A4 inhibitory effect over the inducing potential of etravirine. This observation is in line
Table 3 Summary of amlodipine PK parameters following several dosage regimens, in presence or absence of CYP3A4 inhibitors or efavirenz, derived from model-based simulations. PK values are presented as median ( $95 \%$ prediction interval). $C_{\max }$ maximal concentrations,

\begin{tabular}{llll}
\hline & Standard dosage of 5 mg qd & $2.5 \mathrm{mg}$ qd with CYP3A4 inhibitors & 10 mg qd with efavirenz \\
\hline $\mathrm{C}_{\max }(\mathrm{ng} / \mathrm{mL})$ & $13.6(7.3-29.0)$ & $12.7(6.2-27.8)$ & $13.7(8.4-26.4)$ \\
$\mathrm{GMR}$ & & 0.92 & 1.01 \\
$\mathrm{C}_{\text {trough }}(\mathrm{ng} / \mathrm{mL})$ & $10.2(3.6-25.5)$ & $10.9(4.3-26.0)$ & $6.5(1.5-19.0)$ \\
$\mathrm{GMR}$ & & 1.08 & 0.62 \\
$\mathrm{AUC}_{0-24}(\mathrm{ng} \mathrm{h} / \mathrm{mL})$ & $290.8(129.3-658.4)$ & $285.7(127.0-646.8)$ & $242.3(107.8-548.7)$ \\
$\mathrm{GMR}$ & & 0.98 & 0.83
\end{tabular}

$C_{\text {trough }}$ residual concentrations ( $24 \mathrm{~h}$ after the last drug intake), $A U C_{0-24}$ area under the concentration-time curve from 0 to $24 \mathrm{~h}$ calculated as dose/CL, GMR geometric mean ratio compared with the standard regimen of $5 \mathrm{mg}$ qd 
with current knowledge on the coadministration of CYP3A4 inducers and inhibitors [37-40]. Regarding ARVs for example, a previously published study demonstrated a 3 -fold increase in maraviroc exposure when coadministered with ritonavir-boosted darunavir and etravirine, compared to that obtained under maraviroc alone [41].

Limitations of the present work should be acknowledged. First, CYP3A4 inhibitors and efavirenz were the only covariates showing significant effects, while an effect of age, gender and body weight has been demonstrated in other population pharmacokinetics studies [6, $8,9]$. Yet, the limited number of patients and the sparse sampling schedule for most study data possibly compromise the statistical power of the study to reveal the effects of some covariates. In addition, the parsimonious one-compartment model may have affected the covariate selection [42]. Finally, the small number of PLWH receiving each ARV CYP3A4 inhibitor prevented us not only to establish an interaction model considering ARV drugs plasma concentrations but also to discriminate the effect of individual CYP3A4 inhibitors.

Despite these limitations, the information provided by our analysis on the magnitude of DDIs between amlodipine and ARVs is sufficiently robust to guide clinicians about initial drug dosage adjustments.

In conclusion, our results confirm the half-dosage proposed for amlodipine in case of coadministration of CYP3A4 inhibitors (mainly ritonavir-boosted darunavir), and suggest doubling the dose when amlodipine is coadministered with efavirenz.

Supplementary Information The online version contains supplementary material available at https://doi.org/10.1007/s00228-020-03060-2.

Acknowledgments Members of the Swiss HIV Cohort Study are: AebiPopp K, Anagnostopoulos A, Battegay M, Bernasconi E, Böni J, Braun DL, Bucher HC, Calmy A, Cavassini M, Ciuffi A, Dollenmaier G, Egger M, Elzi L, Fehr J, Fellay J, Furrer H, Fux CA, Günthard HF (President of the SHCS), Haerry D (deputy of "Positive Council"), Hasse B, Hirsch HH, Hoffmann M, Hösli I, Huber M, Kahlert CR (Chairman of the Mother \& Child Substudy), Kaiser L, Keiser O, Klimkait T, Kouyos RD, Kovari H, Ledergerber B, Martinetti G, Martinez de Tejada B, Marzolini C, Metzner KJ, Müller N, Nicca D, Paioni P, Pantaleo G, Perreau M, Rauch A (Chairman of the Scientific Board), Rudin C, Scherrer AU (Head of Data Centre), Schmid P, Speck R, Stöckle M (Chairman of the Clinical and Laboratory Committee), Tarr P, Trkola A, Vernazza P, Wandeler G, Weber R, Yerly S.

Author contribution PC collected the data, performed the population pharmacokinetic analyses, interpreted the data and drafted the manuscript. MG supervised the population pharmacokinetic analyses and reviewed the manuscript. SAS participated in data collection. MC, MS, $\mathrm{TB}$ and $\mathrm{CM}$ brought their clinical expertise to interpret the results and reviewed the manuscript. LAD and TB were the principal investigators of the study proposal and reviewed the manuscript. CC supervised the population pharmacokinetic work.
Funding Open Access funding provided by University of Geneva. This work was supported by two Swiss National Science Foundation grants (grants number 165956 for Lausanne, 166204 for Basel), the OPO Foundation, and the Isaac Dreyfus Foundation (Basel).

Availability of data and material The data that support the findings of this study are available on request from the corresponding author. The data are not publicly available due to privacy or ethical restrictions.

\section{Compliance with ethical standards}

Conflict of interest MC has received through his institution research grant from ViiV, Gilead, and offered expert testimony for Abbvie, MSD, Gilead, and Sandoz. MS received payments for advisory boards from Gilead, ViiV, MSD, Sandoz and Mepha, as well as travel grants for conferences from Gilead and MSD, yet unrelated to the present study. $\mathrm{CM}$ received a research grant from Gilead and speaker honoraria for her institution from MSD. All other authors: none to declare.

Ethics approval The protocol of the study with rich sampling was reviewed and approved by the Ethics Committee of Vaud and northwest/central Switzerland (CER-VD 2018-00369).

Consent to participate Written informed consent was obtained from each patient participating in the study with rich sampling.

Consent for publication Not applicable.

Code availability Not applicable.

Open Access This article is licensed under a Creative Commons Attribution 4.0 International License, which permits use, sharing, adaptation, distribution and reproduction in any medium or format, as long as you give appropriate credit to the original author(s) and the source, provide a link to the Creative Commons licence, and indicate if changes were made. The images or other third party material in this article are included in the article's Creative Commons licence, unless indicated otherwise in a credit line to the material. If material is not included in the article's Creative Commons licence and your intended use is not permitted by statutory regulation or exceeds the permitted use, you will need to obtain permission directly from the copyright holder. To view a copy of this licence, visit http://creativecommons.org/licenses/by/4.0/.

\section{References}

1. Glesby MJ, Aberg JA, Kendall MA, Fichtenbaum CJ, Hafner R, Hall S, Grosskopf N, Zolopa AR, Gerber JG, Adult ACTGAPT (2005) Pharmacokinetic interactions between indinavir plus ritonavir and calcium channel blockers. Clin Pharmacol Ther 78(2):143153. https://doi.org/10.1016/j.clpt.2005.04.005

2. Lee JE, van Heeswijk R, Alves K, Smith F, Garg V (2011) Effect of the hepatitis $\mathrm{C}$ virus protease inhibitor telaprevir on the pharmacokinetics of amlodipine and atorvastatin. Antimicrob Agents Chemother 55(10):4569-4574. https://doi.org/10.1128/AAC. 00653-11

3. Meredith PA, Elliott HL (1992) Clinical pharmacokinetics of amlodipine. Clin Pharmacokinet 22(1):22-31. https://doi.org/10. 2165/00003088-199222010-00003

4. Food and Drug Administration. Norvasc, Summary of Product Characteristics. https://www.accessdata.fda.gov/drugsatfda_docs/ label/2011/019787s047lbl.pdf. Accessed 7 June 2019 
5. Menon RM, Badri PS, Wang T, Polepally AR, Zha J, Khatri A, Wang H, Hu B, Coakley EP, Podsadecki TJ, Awni WM, Dutta S (2015) Drug-drug interaction profile of the all-oral anti-hepatitis C virus regimen of paritaprevir/ritonavir, ombitasvir, and dasabuvir. $\mathrm{J}$ Hepatol 63(1):20-29. https://doi.org/10.1016/j.jhep.2015.01.026

6. Flynn JT, Nahata MC, Mahan JD Jr, Portman RJ, Investigators $P$ (2006) Population pharmacokinetics of amlodipine in hypertensive children and adolescents. J Clin Pharmacol 46(8):905-916. https:// doi.org/10.1177/0091270006289844

7. Heo YA, Holford N, Kim Y, Son M, Park K (2016) Quantitative model for the blood pressure-lowering interaction of valsartan and amlodipine. Br J Clin Pharmacol 82(6):1557-1567. https://doi.org/ 10.1111/bcp. 13082

8. Kang D, Verotta D, Schwartz JB (2006) Population analyses of amlodipine in patients living in the community and patients living in nursing homes. Clin Pharmacol Ther 79(1):114-124. https://doi. org/10.1016/j.clpt.2005.09.007

9. Rohatagi S, Carrothers TJ, Kshirsagar S, Khariton T, Lee J, Salazar D (2008) Evaluation of population pharmacokinetics and exposureresponse relationship with coadministration of amlodipine besylate and olmesartan medoxomil. J Clin Pharmacol 48(7):823-836. https://doi.org/10.1177/0091270008317847

10. Son H, Lee D, Lim LA, Jang SB, Roh H, Park K (2014) Development of a pharmacokinetic interaction model for coadministration of simvastatin and amlodipine. Drug Metab Pharmacokinet 29(2):120-128

11. Cockcroft DW, Gault MH (1976) Prediction of creatinine clearance from serum creatinine. Nephron 16(1):31-41. https://doi.org/10. $1159 / 000180580$

12. Courlet P, Spaggiari D, Desfontaine V, Cavassini M, Alves Saldanha S, Buclin T, Marzolini C, Csajka C, Decosterd LA (2019) UHPLC-MS/MS assay for simultaneous determination of amlodipine, metoprolol, pravastatin, rosuvastatin, atorvastatin with its active metabolites in human plasma, for population-scale drugdrug interactions studies in people living with HIV. J Chromatogr B Anal Technol Biomed Life Sci 1125:121733. https://doi.org/10. 1016/j.jchromb.2019.121733

13. Choi Y, Lee S, Cho SM, Kang WH, Nam KY, Jang IJ, Yu KS (2016) Comparisons of the pharmacokinetics and tolerability of fixed-dose combinations of amlodipine besylate/losartan and amlodipine camsylate/losartan in healthy subjects: a randomized, open-label, single-dose, two-period, two-sequence crossover study. Drug Des Devel Ther 10:3021-3028. https://doi.org/10.2147/ DDDT.S113891

14. Zarghi A, Foroutan SM, Shafaati A, Khoddam A (2005) Validated HPLC method for determination of amlodipine in human plasma and its application to pharmacokinetic studies. Farmaco 60(9):789792. https://doi.org/10.1016/j.farmac.2005.06.012

15. Lindbom L, Pihlgren P, Jonsson EN (2005) PsN-toolkit-a collection of computer intensive statistical methods for non-linear mixed effect modeling using NONMEM. Comput Methods Prog Biomed 79(3):241-257. https://doi.org/10.1016/j.cmpb.2005.04.005

16. Keizer RJ, van Benten M, Beijnen JH, Schellens JH, Huitema AD (2011) Pirana and PCluster: a modeling environment and cluster infrastructure for NONMEM. Comput Methods Prog Biomed 101(1):72-79. https://doi.org/10.1016/j.cmpb.2010.04.018

17. Food and drug administration. Drug development and drug interactions: table of substrates, inhibitors and inducers. https://www.fda. gov/drugs/developmentapprovalprocess/developmentresources/ druginteractionslabeling/ucm093664.htm. Accessed 20 Dec 2018

18. Duffull SB, Wright DF, Winter HR (2011) Interpreting population pharmacokinetic-pharmacodynamic analyses - a clinical viewpoint. Br J Clin Pharmacol 71(6):807-814. https://doi.org/10.1111/j. 1365-2125.2010.03891.x

19. Gab-jin P, Dooyeon J, Jong nyeong K, Sunil Y, Wan-Su P, Jongtae L, Seunghoon H, Dong-Seok Y (2015) Drug-drug interaction analysis of a drug with long elimination half-life using population pharmacokinetic approach. Paper presented at the Population Approach Group in Europe, Hersonissos

20. Suein C, Seunghoon H, Dong-Seok Y (2019) Contribution of trough concentration data for the evaluation of multiple-dose pharmacokinetics. Paper presented at the Population approach group in Europe, Stockholm, 11-14.06.2019

21. Mukherjee D, Zha J, Menon RM, Shebley M (2018) Guiding dose adjustment of amlodipine after co-administration with ritonavir containing regimens using a physiologically-based pharmacokinetic/pharmacodynamic model. J Pharmacokinet Pharmacodyn 45(3):443-456. https://doi.org/10.1007/s10928018-9574-0

22. Stader F, Courlet P, Kinvig H, Penny MA, Decosterd LA, Battegay M, Siccardi M, Marzolini C (2020) Clinical data combined with modelling and simulation indicate unchanged drug-drug interaction magnitudes in the elderly. Clin Pharmacol Ther. https://doi.org/10. 1002/cpt.2017

23. Bleyzac N, Bourguignon L, Goutelle S, Tod MDDI. Impact of drug-drug interactions of drug exposure https://www.ddipredictor.org/predictor/ddi

24. Stader F, Kinvig H, Battegay M, Khoo S, Owen A, Siccardi M, Marzolini C (2018) Analysis of clinical drug-drug interaction data to predict magnitudes of uncharacterized interactions between antiretroviral drugs and comedications. Antimicrob Agents Chemother 62(7):e00717-e00718. https://doi.org/10.1128/AAC.00717-18

25. Marzolini C, Gibbons S, Khoo S, Back D (2016) Cobicistat versus ritonavir boosting and differences in the drug-drug interaction profiles with co-medications. J Antimicrob Chemother 71(7):17551758. https://doi.org/10.1093/jac/dkw032

26. Liverpool HIV drug interactions website. http://www.hivdruginteractions.org/. Accessed 16.07.2019

27. Izzedine H, Launay-Vacher V, Deray G, Hulot JS (2004) Nelfinavir and felodipine: a cytochrome P450 3A4-mediated drug interaction. Clin Pharmacol Ther 75(4):362-363. https://doi.org/10.1016/j.clpt. 2003.12.006

28. Puech R, Gagnieu MC, Planus C, Charpiat B, Boibieux A, Ferry T, Tod M (2011) Extreme bradycardia due to multiple drug-drug interactions in a patient with HIV post-exposure prophylaxis containing lopinavir-ritonavir. Br J Clin Pharmacol 71(4):621-623. https:// doi.org/10.1111/j.1365-2125.2010.03849.x

29. Baeza MT, Merino E, Boix V, Climent E (2007) Nifedipinelopinavir/ritonavir severe interaction: a case report. Aids 21(1): 119-120. https://doi.org/10.1097/QAD.0b013e3280117f6f

30. Kader C, Ede H, Erbay A, Erbay A (2015) Drug interaction of boceprevir and amlodipine in a patient with hepatitis C: a cardiovascular follow-up. J Microbiol Infect Dis 5(1):32-35. https://doi. org/10.5799/ahinjs.02.2015.01.0172

31. Molto J, Rajoli R, Back D, Valle M, Miranda C, Owen A, Clotet B, Siccardi M (2017) Use of a physiologically based pharmacokinetic model to simulate drug-drug interactions between antineoplastic and antiretroviral drugs. J Antimicrob Chemother 72(3):805-811. https://doi.org/10.1093/jac/dkw485

32. Kapetas AJ, Sorich MJ, Rodrigues AD, Rowland A (2019) Guidance for rifampin and midazolam dosing protocols to study intestinal and hepatic cytochrome P450 (CYP) 3A4 induction and De-induction. AAPS J 21(5):78. https://doi.org/10.1208/s12248019-0341-y

33. Leenen FH, Coletta E (2010) Pharmacokinetic and antihypertensive profile of amlodipine and felodipine-ER in younger versus older patients with hypertension. J Cardiovasc Pharmacol 56(6):669 675. https://doi.org/10.1097/FJC.0b013e3181fc45bb

34. Flexner C, Tierney C, Gross R, Andrade A, Lalama C, Eshleman SH, Aberg J, Sanne I, Parsons T, Kashuba A, Rosenkranz SL, Kmack A, Ferguson E, Dehlinger M, Mildvan D, Team AAS (2010) Comparison of once-daily versus twice-daily combination 
antiretroviral therapy in treatment-naive patients: results of AIDS clinical trials group (ACTG) A5073, a 48-week randomized controlled trial. Clin Infect Dis 50(7):1041-1052. https://doi.org/10. 1086/651118

35. Kamal S, Bugnon O, Cavassini M, Schneider MP (2018) HIVinfected patients' beliefs about their chronic co-treatments in comparison with their combined antiretroviral therapy. HIV Med 19(1): 49-58. https://doi.org/10.1111/hiv.12542

36. Song I, Borland J, Min S, Lou Y, Chen S, Patel P, Wajima T, Piscitelli SC (2011) Effects of etravirine alone and with ritonavirboosted protease inhibitors on the pharmacokinetics of dolutegravir. Antimicrob Agents Chemother 55(7):3517-3521. https://doi.org/10.1128/AAC.00073-11

37. Hafner V, Jager M, Matthee AK, Ding R, Burhenne J, Haefeli WE, Mikus G (2010) Effect of simultaneous induction and inhibition of CYP3A by St John's Wort and ritonavir on CYP3A activity. Clin Pharmacol Ther 87(2):191-196. https://doi.org/10.1038/clpt.2009. 206

38. Duval X, Le Moing V, Longuet C, Leport C, Vilde JL, Lamotte C, Peytavin G, Farinotti R (2000) Efavirenz-induced decrease in plasma amprenavir levels in human immunodeficiency virus-infected patients and correction by ritonavir. Antimicrob Agents Chemother 44(9):2593. https://doi.org/10.1128/aac.44.9.2593-2593.2000
39. Abel S, Jenkins TM, Whitlock LA, Ridgway CE, Muirhead GJ (2008) Effects of CYP3A4 inducers with and without CYP3A4 inhibitors on the pharmacokinetics of maraviroc in healthy volunteers. Br J Clin Pharmacol 65(Suppl 1):38-46. https://doi.org/10. 1111/j.1365-2125.2008.03134.x

40. Kakuda TN, Abel S, Davis J, Hamlin J, Scholler-Gyure M, Mack R, Ndongo N, Petit W, Ridgway C, Sekar V, Tweedy S, Hoetelmans RM (2011) Pharmacokinetic interactions of maraviroc with darunavir-ritonavir, etravirine, and etravirine-darunavir-ritonavir in healthy volunteers: results of two drug interaction trials. Antimicrob Agents Chemother 55(5):2290-2296. https://doi.org/ 10.1128/AAC.01046-10

41. Wade JR, Beal SL, Sambol NC (1994) Interaction between structural, statistical, and covariate models in population pharmacokinetic analysis. J Pharmacokinet Biopharm 22(2):165-177. https://doi. org/10.1007/bf02353542

42. Tseng A, Hughes CA, Wu J, Seet J, Phillips EJ (2017) Cobicistat versus ritonavir: similar pharmacokinetic enhancers but some important differences. Ann Pharmacother 51(11):1008-1022. https:// doi.org/10.1177/1060028017717018

Publisher's note Springer Nature remains neutral with regard to jurisdictional claims in published maps and institutional affiliations. 